\title{
Summation process of Korovkin type approximation theorem
}

Sevda Karakus and Kamil Demirci 


\title{
SUMMATION PROCESS OF KOROVKIN TYPE APPROXIMATION THEOREM
}

\author{
SEVDA KARAKUŞ AND KAMIL DEMIRCI
}

Received 30 March, 2010

\begin{abstract}
The aim of this paper is to present a Korovkin-type approximation theorem on $H_{w}\left(I^{2}\right)$, which is the subspace of all continuous and bounded real valued functions on $I^{2}=[0, \infty) \times$ $[0, \infty)$ by using $\mathcal{A}$-summation process.
\end{abstract}

2000 Mathematics Subject Classification: 40G15; 41A36

Keywords: matrix summability, positive linear operators, bivariate Korovkin theorem, Bleimann, Butzer and Hahn operators

\section{INTRODUCTION}

Approximation theory, which has a close relationship with other branches of mathematics, has been used in the theory of polynomial approximation and various domains of functional analysis [2], in numerical studies of differential and integral operators [15], and in the studies of the interpolation operator of Hermite-Fejér [6-8,10] and of the partial sums of Fourier series [16]. Most of the classical approximation operators tend to converge to the value of the function being approximated. However, at points of discontinuity, they often converge to the average of the left and right limits of the function. There are, however, exceptions such as the interpolation operators of Hermite-Fejér [6]. These operators do not converge at points of simple discontinuity. In this case, the matrix summability methods of Cesáro type are strong enough to correct the lack of convergence [7]. The main purpose of using summability theory has always been to make a nonconvergent sequence converge. Some results regarding matrix summability for positive linear operators may be found in the papers $[3,4,14,23]$. Our interest in the present paper is to obtain a Korovkintype approximation theorem for a sequence of positive linear operators defined on $H_{w}\left(I^{2}\right)$, which is the subspace of all continuous and bounded real valued functions on $I^{2}=[0, \infty) \times[0, \infty)$ by using $\mathcal{A}$-summation process.

A double sequence $x=\left\{x_{m, n}\right\}_{m, n \in \mathbb{N}}$ is convergent in Pringsheim's sense if, for every $\varepsilon>0$, there exists $N=N(\varepsilon) \in \mathbb{N}$ such that $\left|x_{m, n}-L\right|<\varepsilon$ whenever $m, n>N$. 
In this case $L$ is called the Pringsheim limit of $x$ and is denoted by $P-\lim x=L$ (see [22]).

If there exists a positive number $M$ such that $\left|x_{m, n}\right| \leq M$ for all $(m, n) \in \mathbb{N}^{2}=$ $\mathbb{N} \times \mathbb{N}$, then $x=\left\{x_{m, n}\right\}$ is said to be bounded. Note that in contrast to the case for single sequences, a convergent double sequence need not to be bounded.

Let

$$
A=\left[a_{j, k, m, n}\right], \quad j, k, m, n \in \mathbb{N},
$$

be a four-dimensional infinite matrix. For a given double sequence $x=\left\{x_{m, n}\right\}$, the $A$-transform of $x$, denoted by $A x:=\left\{(A x)_{j, k}\right\}$, is given by

$$
(A x)_{j, k}=\sum_{(m, n) \in \mathbb{N}^{2}} a_{j, k, m, n} x_{m, n}, \quad j, k \in \mathbb{N},
$$

provided the double series converges in Pringsheim's sense for every $(j, k) \in \mathbb{N}^{2}$. We say that a sequence $x$ is $A$-summable to $l$ if the $A$-transform of $x$ exists for all $j, k \in \mathbb{N}$ and is convergent in the Pringsheim's sense i.e.,

$$
P-\lim _{p, q} \sum_{m \in \mathbb{N}}^{p} \sum_{n \in \mathbb{N}}^{q} a_{j, k, m, n} x_{m, n}=y_{j, k} \text { and } P-\lim _{j, k} y_{j, k}=l .
$$

In summability theory, a two-dimensional matrix transformation is said to be regular if it maps every convergent sequence to a convergent sequence with the same limit. The well-known characterization of regularity for two dimensional matrix transformations is known as Silverman-Toeplitz conditions (see, for instance, [13]). In 1926, Robison [24] presented a four dimensional analog of the regularity by considering an additional assumption of boundedness. This assumption was made because a double $P$-convergent sequence is not necessarily bounded. The definition and the characterization of regularity for four dimensional matrices is known as Robison-Hamilton conditions, or briefly, $R H$-regularity. (see, [12,24])

Recall that a four dimensional matrix $A=\left[a_{j, k, m, n}\right]$ is said to be $R H$-regular if it maps every bounded $P$-convergent sequence into a $P$-convergent sequence with the same $P$-limit. The Robison-Hamilton conditions state that a four dimensional matrix $A=\left[a_{j, k, m, n}\right]$ is $R H$-regular if and only if

$$
\begin{aligned}
& \text { (i) } P-\lim _{j, k} a_{j, k, m, n}=0 \text { for each }(m, n) \in \mathbb{N}^{2}, \\
& \text { (ii) } P-\lim _{j, k} \sum_{(m, n) \in \mathbb{N}^{2}} a_{j, k, m, n}=1, \\
& \text { (iii) } P-\lim _{j, k} \sum_{m \in \mathbb{N}}\left|a_{j, k, m, n}\right|=0 \text { for each } n \in \mathbb{N}, \\
& \text { (iv) } P-\lim _{j, k} \sum_{n \in \mathbb{N}}\left|a_{j, k, m, n}\right|=0 \text { for each } m \in \mathbb{N},
\end{aligned}
$$


(v) $\sum_{(m, n) \in \mathbb{N}^{2}}\left|a_{j, k, m, n}\right|$ is $P$-convergent for each $(j, k) \in \mathbb{N}^{2}$,

(vi) there exist finite positive integers $A$ and $B$ such that $\sum_{m, n>B}\left|a_{j, k, m, n}\right|<A$ holds for every $(j, k) \in \mathbb{N}^{2}$.

Now let $\mathcal{A}:=\left\{A^{(i, l)}\right\}=\left\{a_{j, k, m, n}^{(i, l)}\right\}$ be a sequence of four-dimensional infinite matrices with non-negative real entries. For a given double sequence of real numbers, $x=\left\{x_{m, n}\right\}$ is said to be $\mathcal{A}$-summable to $l$ if

$$
P-\lim _{j, k} \sum_{(m, n) \in \mathbb{N}^{2}} a_{j, k, m, n}^{(i, l)} x_{m, n}=l
$$

uniformly in $i$ and $l$. If $A^{(i, l)}=A$, four-dimensional infinite matrix, then $\mathcal{A}$-summability is the $A$-summability for four-dimensional infinite matrix.

Some results regarding matrix summability method for double sequences may be found in the papers [20], [21], [25].

Now let $A=\left[a_{j, k, m, n}\right]$ be a non-negative $R H$-regular summability matrix, and let $K \subset \mathbb{N}^{2}$. Then, a real double sequence $x=\left\{x_{m, n}\right\}$ is said to be $A$-statistically convergent to a number $L$ if, for every $\varepsilon>0$,

$$
P-\lim _{j, k} \sum_{(m, n) \in K(\varepsilon)} a_{j, k, m, n}=0,
$$

where

$$
K(\varepsilon):=\left\{(m, n) \in \text { BeginExpansion } \mathbb{N}^{2}:\left|x_{m, n}-L\right| \geq \varepsilon\right\} .
$$

In this case we write $s t_{A}^{2}-\lim _{m, n} x_{m, n}=L$. Observe that, a $P$-convergent double sequence is $A$-statistically convergent to the same value but the converse does not hold true.

We should note that if we take $A=C(1,1)$, which is the double Cesáro matrix, then $C(1,1)$-statistical convergence coincides with the notion of statistical convergence for double sequence, which was introduced in [18, 19]. Finally, if we replace the matrix $A$ by the identity matrix for four-dimensional matrices, then $A$-statistical convergence reduces to the Pringsheim convergence.

\section{A Korovkin-TyPe APPROXIMATION THEOREM}

We recall that the Korovkin-type theorem on the $H_{w}([0, \infty))$ space was given by Gadjiev and Çakar in [11]. Similarly as in [11], let us introduce a space denoted by $H_{w}\left(I^{2}\right)$, where $I^{2}:=[0, \infty) \times[0, \infty)$.

Let $\omega$ be a modulus of continuity type functions such that the following conditions are satisfied:

i) $\omega$ is a non-negative increasing function on $[0, \infty)$, 
ii) $\omega\left(\delta_{1}+\delta_{2}\right) \leq \omega\left(\delta_{1}\right)+\omega\left(\delta_{2}\right)$,

iii) $\lim _{\delta \rightarrow 0} \omega(\delta)=0$.

Let $H_{\omega}\left(I^{2}\right)$ be a subspace of real valued functions satisfying the following conditions: for some $M>0$

$$
|f(u, v)-f(x, y)| \leq M \omega\left(\left|\left(\frac{u}{1+u}, \frac{v}{1+v}\right)-\left(\frac{x}{1+x}, \frac{y}{1+y}\right)\right|\right)
$$

where

$\left|\left(\frac{u}{1+u}, \frac{v}{1+v}\right)-\left(\frac{x}{1+x}, \frac{y}{1+y}\right)\right|=\sqrt{\left(\frac{u}{1+u}-\frac{x}{1+x}\right)^{2}+\left(\frac{v}{1+v}-\frac{y}{1+y}\right)^{2}}$.

Let $C_{B}\left(I^{2}\right)$ be the space of all continuous and bounded functions on $I^{2}$. Then $C_{B}\left(I^{2}\right)$ is a linear normed space with

$$
\|f\|_{C_{B}\left(I^{2}\right)}=\sup _{x, y \geq 0}|f(x, y)| .
$$

Due to (ii), we can say that $H_{\omega}\left(I^{2}\right) \subset C_{B}\left(I^{2}\right)$.

A sequence $\left\{L_{m, n}\right\}$ of positive linear operators of $H_{\omega}\left(I^{2}\right)$ into $C_{B}\left(I^{2}\right)$ is called an $\mathcal{A}$-summation process on $H_{\omega}\left(I^{2}\right)$ if $\left\{L_{m, n}(f)\right\}$ is $\mathcal{A}$-summable to $f$ for every $f \in H_{\omega}\left(I^{2}\right)$, i.e.,

$$
P-\lim _{j, k \rightarrow \infty}\left\|\sum_{(m, n) \in \mathbb{N}^{2}} a_{j, k, m, n}^{(i, l)} L_{m, n}(f)-f\right\|_{C_{B}\left(I^{2}\right)}=0, \quad \text { uniformly in } i \text { and } l
$$

where it is assumed that the series in (2.2) converges for each $i, l, j, k \in \mathbb{N}$ and $f$.

Note that, the results of type (2.2) are extensions of type

$$
P-\lim _{j, k \rightarrow \infty} \sum_{(m, n) \in \mathbb{N}^{2}} a_{j, k, m, n}^{(i, l)}\left\|L_{m, n}(f)-f\right\|_{C_{B}\left(I^{2}\right)}=0, \quad \text { uniformly in } i \text { and } l
$$

for all $f \in H_{\omega}\left(I^{2}\right)$.

We establish a theorem of Korovkin type with respect to the convergence behavior (2.2) for a double sequence of positive linear operators of $H_{\omega}\left(I^{2}\right)$ into $C_{B}\left(I^{2}\right)$.

Let $\left\{L_{m, n}\right\}$ be a sequence of positive linear operators of $H_{\omega}\left(I^{2}\right)$ into $C_{B}\left(I^{2}\right)$ such that

$$
\sup _{i, l, j, k} \sum_{(m, n) \in \mathbb{N}^{2}} a_{j, k, m, n}^{(i, l)}\left\|L_{m, n}(1)\right\|_{C_{B}\left(I^{2}\right)}<\infty .
$$

Furthermore, for $i, l, j, k \in \mathbb{N}$ and $f \in H_{\omega}\left(I^{2}\right)$, let

$$
B_{j, k}^{(i, l)} f=\sum_{(m, n) \in \mathbb{N}^{2}} a_{j, k, m, n}^{(i, l)} L_{m, n}(f)
$$


which is well defined by (2.3) and belongs to $C_{B}\left(I^{2}\right)$.

Altın, Doğru and Özarslan [1] obtained the following Korovkin theorem.

Theorem 1 ([1]). Let $\left\{L_{m, n}\right\}$ be a double sequence of positive linear operators acting from $H_{\omega}\left(I^{2}\right)$ into $C_{B}\left(I^{2}\right)$. Then, for all $f \in H_{\omega}\left(I^{2}\right)$

$$
P-\lim _{m, n}\left\|L_{m, n}(f)-f\right\|_{C_{B}\left(I^{2}\right)}=0
$$

is satisfied if the following holds:

$$
P-\lim _{m, n}\left\|L_{m, n}\left(f_{i}\right)-f_{i}\right\|_{C_{B}\left(I^{2}\right)}=0 \quad(i=0,1,2,3)
$$

where

$$
\begin{gathered}
f_{0}(u, v)=1, \quad f_{1}(u, v)=\frac{u}{1+u}, \quad f_{2}(u, v)=\frac{v}{1+v}, \\
f_{3}(u, v)=\left(\frac{u}{1+u}\right)^{2}+\left(\frac{v}{1+v}\right)^{2} .
\end{gathered}
$$

$A$-statistical analog of Theorem 1 can be given as follows.

Theorem 2 ([9]). Let $A=\left[a_{j, k, m, n}\right]$ be a non-negative $R H$-regular summability matrix method. Let $\left\{L_{m, n}\right\}$ be a double sequence of positive linear operators acting from $H_{\omega}\left(I^{2}\right)$ into $C_{B}\left(I^{2}\right)$. Then, for all $f \in H_{\omega}\left(I^{2}\right)$

$$
s t_{A}^{2}-\lim _{m, n}\left\|L_{m, n}(f)-f\right\|_{C_{B}\left(I^{2}\right)}=0
$$

is satisfied if the following holds:

$$
s t_{A}^{2}-\lim _{m, n}\left\|L_{m, n}\left(f_{i}\right)-f_{i}\right\|_{C_{B}\left(I^{2}\right)}=0 \quad(i=0,1,2,3)
$$

where

$$
\begin{gathered}
f_{0}(u, v)=1, \quad f_{1}(u, v)=\frac{u}{1+u}, \quad f_{2}(u, v)=\frac{v}{1+v}, \\
f_{3}(u, v)=\left(\frac{u}{1+u}\right)^{2}+\left(\frac{v}{1+v}\right)^{2} .
\end{gathered}
$$

If we replace the matrix $A$ in Theorem 2 by the Cesáro matrix $C(1,1)$, we immediately get the statistical Korovkin result.

Now we give the following generalization by using a $\mathcal{A}$-summation process.

Theorem 3. Let $\mathcal{A}=\left\{A^{(i, l)}\right\}$ be a sequence of four-dimensional infinite matrices with non-negative real entries such that

$$
\sup _{i, l, j, k} \sum_{(m, n) \in \mathbb{N}^{2}} a_{j, k, m, n}^{(i, l)}<\infty
$$


Let $\left\{L_{m, n}\right\}$ be a double sequence of positive linear operators acting from $H_{\omega}\left(I^{2}\right)$ into $C_{B}\left(I^{2}\right)$. Assume that (2.3) holds. Then, for all $f \in H_{\omega}\left(I^{2}\right)$

$$
P-\lim _{j, k}\left\|\sum_{(m, n) \in \mathbb{N}^{2}} a_{j, k, m, n}^{(i, l)} L_{m, n}(f)-f\right\|_{C_{B}\left(I^{2}\right)}=0 \text { uniformly in } i \text { and } l
$$

is satisfied if the following holds:

$$
P-\lim _{j, k}\left\|\sum_{(m, n) \in \mathbb{N}^{2}} a_{j, k, m, n}^{(i, l)} L_{m, n}\left(f_{r}\right)-f_{r}\right\|_{C_{B}\left(I^{2}\right)}=0
$$

uniformly in $i$ and $l(r=0,1,2,3)$, where

$$
\begin{gathered}
f_{0}(u, v)=1, \quad f_{1}(u, v)=\frac{u}{1+u}, \quad f_{2}(u, v)=\frac{v}{1+v}, \\
f_{3}(u, v)=\left(\frac{u}{1+u}\right)^{2}+\left(\frac{v}{1+v}\right)^{2} .
\end{gathered}
$$

Proof. If $f \in H_{\omega}\left(I^{2}\right)$, then from (2.1) we have that for any $\varepsilon>0$ there exists a number $\delta>0$ such that $|f(u, v)-f(x, y)|<\varepsilon$ if

$$
\sqrt{\left(\frac{u}{1+u}-\frac{x}{1+x}\right)^{2}+\left(\frac{v}{1+v}-\frac{y}{1+y}\right)^{2}}<\delta .
$$

Since $f$ is bounded, there exists a positive constant $N$ such that

$$
|f(u, v)-f(x, y)|<\frac{2 N}{\delta^{2}}\left[\left(\frac{u}{1+u}-\frac{x}{1+x}\right)^{2}+\left(\frac{v}{1+v}-\frac{y}{1+y}\right)^{2}\right]
$$

if $\sqrt{\left(\frac{u}{1+u}-\frac{x}{1+x}\right)^{2}+\left(\frac{v}{1+v}-\frac{y}{1+y}\right)^{2}} \geq \delta$. Therefore for all $(u, v),(x, y) \in I^{2}$ one can write

$$
|f(u, v)-f(x, y)|<\varepsilon+\frac{2 N}{\delta^{2}}\left[\left(\frac{u}{1+u}-\frac{x}{1+x}\right)^{2}+\left(\frac{v}{1+v}-\frac{y}{1+y}\right)^{2}\right] .
$$

Now using the linearity and the positivity of the operators $L_{m, n}$ and considering the inequalites (2.6) and (2.4), for all $(x, y) \in I^{2}$, we obtain

$$
\begin{aligned}
& \left|\sum_{(m, n) \in \mathbb{N}^{2}} a_{j, k, m, n}^{(i, l)} L_{m, n}(f ; x, y)-f(x, y)\right| \\
\leq & \sum_{(m, n) \in \mathbb{N}^{2}} a_{j, k, m, n}^{(i, l)} L_{m, n}(|f(u, v)-f(x, y)| ; x, y)
\end{aligned}
$$




$$
\begin{aligned}
& +|f(x, y)|\left|\sum_{(m, n) \in \mathbb{N}^{2}} a_{j, k, m, n}^{(i, l)} L_{m, n}(1 ; x, y)-1\right| \\
& \leq \sum_{(m, n) \in \mathbb{N}^{2}} a_{j, k, m, n}^{(i, l)} L_{m, n}\left(\varepsilon+\frac{2 N}{\delta^{2}}\left[\left(\frac{u}{1+u}-\frac{x}{1+x}\right)^{2}+\left(\frac{v}{1+v}-\frac{y}{1+y}\right)^{2}\right] ; x, y\right) \\
& +N\left|\sum_{(m, n) \in \mathbb{N}^{2}} a_{j, k, m, n}^{(i, l)} L_{m, n}(1 ; x, y)-1\right| \\
& \leq \varepsilon+\varepsilon\left|\sum_{(m, n) \in \mathbb{N}^{2}} a_{j, k, m, n}^{(i, l)} L_{m, n}(1 ; x, y)-1\right|+N\left|\sum_{(m, n) \in \mathbb{N}^{2}} a_{j, k, m, n}^{(i, l)} L_{m, n}(1 ; x, y)-1\right| \\
& +\frac{2 N}{\delta^{2}} \sum_{(m, n) \in \mathbb{N}^{2}} a_{j, k, m, n}^{(i, l)} L_{m, n}\left(\left(\frac{u}{1+u}-\frac{x}{1+x}\right)^{2} ; x, y\right) \\
& +\frac{2 N}{\delta^{2}} \sum_{(m, n) \in \mathbb{N}^{2}} a_{j, k, m, n}^{(i, l)} L_{m, n}\left(\left(\frac{v}{1+v}-\frac{v}{1+v}\right)^{2} ; x, y\right) \\
& \leq \varepsilon+\left(\varepsilon+N+\frac{4 N}{\delta^{2}}\right)\left|\sum_{(m, n) \in \mathbb{N}^{2}} a_{j, k, m, n}^{(i, l)} L_{m, n}(1 ; x, y)-1\right| \\
& +\frac{2 N}{\delta^{2}} \mid \sum_{(m, n) \in \mathbb{N}^{2}} a_{j, k, m, n}^{(i, l)} L_{m, n}\left(\left(\frac{u}{1+u}\right)^{2}+\left(\frac{v}{1+v}\right)^{2} ; x, y\right) \\
& -\left(\left(\frac{x}{1+x}\right)^{2}+\left(\frac{y}{1+y}\right)^{2}\right) \\
& +\frac{4 N}{\delta^{2}}\left|\sum_{(m, n) \in \mathbb{N}^{2}} a_{j, k, m, n}^{(i, l)} L_{m, n}\left(\left(\frac{u}{1+u}\right) ; x, y\right)-\frac{x}{1+x}\right| \\
& +\frac{4 N}{\delta^{2}}\left|\sum_{(m, n) \in \mathbb{N}^{2}} a_{j, k, m, n}^{(i, l)} L_{m, n}\left(\left(\frac{v}{1+v}\right) ; x, y\right)-\frac{y}{1+y}\right| \\
& \leq \varepsilon+B\left\{\left|\sum_{(m, n) \in \mathbb{N}^{2}} a_{j, k, m, n}^{(i, l)} L_{m, n}(1 ; x, y)-1\right|\right.
\end{aligned}
$$




$$
\begin{gathered}
+\left|\sum_{(m, n) \in \mathbb{N}^{2}} a_{j, k, m, n}^{(i, l)} L_{m, n}\left(\left(\frac{u}{1+u}\right) ; x, y\right)-\frac{x}{1+x}\right| \\
+\left|\sum_{(m, n) \in \mathbb{N}^{2}} a_{j, k, m, n}^{(i, l)} L_{m, n}\left(\left(\frac{v}{1+v}\right) ; x, y\right)-\frac{y}{1+y}\right| \\
+\mid \sum_{(m, n) \in \mathbb{N}^{2}} a_{j, k, m, n}^{(i, l)} L_{m, n}\left(\left(\frac{u}{1+u}\right)^{2}+\left(\frac{v}{1+v}\right)^{2} ; x, y\right) \\
\left.-\left(\left(\frac{x}{1+x}\right)^{2}+\left(\frac{y}{1+y}\right)^{2}\right) \mid\right\}
\end{gathered}
$$

where $B:=\max \left\{\varepsilon+N+\frac{4 N}{\delta^{2}}, \frac{2 N}{\delta^{2}}, \frac{4 N}{\delta^{2}}\right\}$. Then taking supremum over $(x, y) \in I^{2}$, we have

$$
\begin{aligned}
& \left\|\sum_{(m, n) \in \mathbb{N}^{2}} a_{j, k, m, n}^{(i, l)} L_{m, n}(f)-f\right\|_{C_{B}\left(I^{2}\right)} \\
& \leq \varepsilon+B\left\{\left\|\sum_{(m, n) \in \mathbb{N}^{2}} a_{j, k, m, n}^{(i, l)} L_{m, n}\left(f_{0}\right)-f_{0}\right\|_{C_{B}\left(I^{2}\right)}\right. \\
& +\left\|\sum_{(m, n) \in \mathbb{N}^{2}} a_{j, k, m, n}^{(i, l)} L_{m, n}\left(f_{1}\right)-f_{1}\right\|_{C_{B}\left(I^{2}\right)} \\
& +\left\|\sum_{(m, n) \in \mathbb{N}^{2}} a_{j, k, m, n}^{(i, l)} L_{m, n}\left(f_{2}\right)-f_{2}\right\|_{C_{B}\left(I^{2}\right)} \\
& \left.+\left\|\sum_{(m, n) \in \mathbb{N}^{2}} a_{j, k, m, n}^{(i, l)} L_{m, n}\left(f_{3}\right)-f_{3}\right\|_{C_{B}\left(I^{2}\right)}\right\} .
\end{aligned}
$$

Using (2.5) and by taking limit as $j, k \rightarrow \infty$, we obtain the desired result.

Remark 1 . If we take $A^{(i, l)}=I, I$ being the four-dimensional identity matrix in Theorem 3, then we immediately get Theorem 1 . 
Corollary 1. Now we present an example such that our new approximation result works but its classical case does not work. Let $I^{2}=[0, \infty) \times[0, \infty)$ and $f: I^{2} \rightarrow \mathbb{R}$. We consider the double sequence $\left\{L_{m, n}\right\}$ of positive linear operators defined by

$$
L_{m, n}(f ; x, y)=\left(1+\alpha_{m, n}\right) B_{m, n}(f ; x, y)
$$

where $\left\{B_{m, n}\right\}$ is the Bleimann, Butzer and Hahn [5] operators defined by

$$
=\frac{1}{(1+x)^{m}} \frac{1}{(1+y)^{n}} \sum_{k=0}^{m} \sum_{l=0}^{n} f\left(\frac{k}{m-k+1}, \frac{l}{n-l+1}\right)\left(\begin{array}{c}
m \\
k
\end{array}\right)\left(\begin{array}{l}
n \\
l
\end{array}\right) x^{k} y^{l}
$$

and $\left(\alpha_{m, n}\right)$ is a double sequence defined by $\alpha_{m, n}=(-1)^{m+n}$.

From [1], we have

$$
\begin{gathered}
B_{m, n}\left(f_{0} ; x, y\right)=1, \\
B_{m, n}\left(f_{1} ; x, y\right)=\frac{m}{m+1} \frac{x}{1+x}, \\
B_{m, n}\left(f_{2} ; x, y\right)=\frac{n}{n+1} \frac{y}{1+y}, \\
B_{m, n}\left(f_{3} ; x, y\right)=\frac{m(m-1)}{(m+1)^{2}} \frac{x^{2}}{(1+x)^{2}}+\frac{m}{(m+1)^{2}} \frac{x}{1+x} \\
+\frac{n(n-1) g}{(n+1)^{2}} \frac{y^{2}}{(1+y)^{2}}+\frac{n}{(n+1)^{2}} \frac{y}{1+y},
\end{gathered}
$$

where

$$
\begin{gathered}
f_{0}(u, v)=1, f_{1}(u, v)=\frac{u}{1+u}, \quad f_{2}(u, v)=\frac{v}{1+v}, \\
f_{3}(u, v)=\left(\frac{u}{1+u}\right)^{2}+\left(\frac{v}{1+v}\right)^{2} .
\end{gathered}
$$

A double sequence $x=\left\{x_{m, n}\right\}$ of real numbers is called almost convergent to a limit $s$ if

$$
P-\lim _{p, q \rightarrow \infty} \sup _{j, k \geq 0}\left|\frac{1}{p q} \sum_{m=j}^{j+p-1} \sum_{n=k}^{k+q-1} x_{m, n}-s\right|=0,
$$

that is, the average value of $\left\{x_{m, n}\right\}$ taken over any rectangle

$$
\{(m, n): j \leq m \leq j+p-1, k \leq n \leq k+q-1\}
$$

tends to $s$ as both $p$ and $q$ tend to $\infty$, and this convergence is uniform in $j$ and $k$. Now assume that $\mathcal{A}=\left\{A^{(i, l)}\right\}=\left\{a_{j, k, m, n}^{(i, l)}\right\}$ is a sequence of four-dimensional infinite matrices defined by $a_{j, k, m, n}^{(i, l)}=\frac{1}{j k}$ if $i \leq m \leq j+i-1, l \leq n \leq k+l-1$ 
and $a_{j, k, m, n}^{(i, l)}=0$ otherwise. In this case $\mathcal{A}$-summability method reduces to almost convergence of double sequences introduced by Moricz [17]. Observe that $\left(\alpha_{m, n}\right)$ is almost convergent to zero, but it is not convergent in Pringsheim's sense. Also $\left(\alpha_{m, n}\right)$ is not C(1,1)-statistically convergent. We conclude that for the double sequence $\left\{L_{m, n}\right\}$, since $\left(\alpha_{m, n}\right)$ is almost convergent to zero, $\left\{L_{m, n}\right\}$ satisfies the conditions of Theorem 3. Also, since $\left(\alpha_{m, n}\right)$ is not convergent in Pringsheim's sense and $C(1,1)$ statistical sense, $\left\{L_{m, n}\right\}$ does not satisfy Theorem 1 and Theorem $2($ for $A=C(1,1)$ ).

\section{REFERENCES}

[1] A. Altın, O. Doğru, and M. A. Özarslan, "Korovkin type approximation properties of bivariate Bleimann, Butzer and Hahn operators," Proceedings of the 8th WSEAS International Conference on APPLIED MATHEMATICS, Tenerife, Spain, December 16-18, pp. 234-238, 2005.

[2] F. Altomare and M. Campiti, Korovkin-type approximation theory and its applications, ser. de Gruyter Studies in Mathematics. Berlin: Walter de Gruyter \& Co., 1994, vol. 17.

[3] O. G. Atlihan and C. Orhan, "Summation process of positive linear operators," Comput. Math. Appl., vol. 56, no. 5, pp. 1188-1195, 2008.

[4] O. G. Atlıhan and C. Orhan, "Matrix summability and positive linear operators," Positivity, vol. 11, no. 3, pp. 387-398, 2007.

[5] G. Bleimann, P. L. Butzer, and L. Hahn, "A Bernšteřn-type operator approximating continuous functions on the semi-axis," Nederl. Akad. Wetensch. Indag. Math., vol. 42, no. 3, pp. 255-262, 1980.

[6] R. Bojanić and F. H. Chêng, "Estimates for the rate of approximation of functions of bounded variation by Hermite-Fejér polynomials," in Second Edmonton conference on approximation theory (Edmonton, Alta., 1982), ser. CMS Conf. Proc., vol. 3. Providence, RI: Amer. Math. Soc., 1983, pp. 5-17.

[7] R. Bojanic and M. K. Khan, "Summability of Hermite-Fejér interpolation for functions of bounded variation," J. Nat. Sci. Math., vol. 32, no. 1, pp. 5-10, 1992.

[8] E. W. Cheney, Introduction to approximation theory. Providence, RI: AMS Chelsea Publishing, 1998, reprint of the second (1982) edition.

[9] K. Demirci and S. Karakuş, "Extension of the Korovkin type approximation theorem in $a$ statistical sense," (submitted), (submitted).

[10] R. A. DeVore, The approximation of continuous functions by positive linear operators, ser. Lecture Notes in Mathematics. Berlin-New York: Springer-Verlag, 1972, vol. 293.

[11] A. D. Gadjiev and O. Çakar, "On uniform approximation by Bleimann, Butzer and Hahn operators on all positive semiaxis," Trans. Acad. Sci. Azerb. Ser. Phys.-Tech. Math. Sci., vol. 19, no. 5, pp. 21-26, 1999.

[12] H. J. Hamilton, “Transformations of multiple sequences,” Duke Math. J., vol. 2, no. 1, pp. 29-60, 1936.

[13] G. H. Hardy, Divergent Series. London: Oxford Univ. Press, 1949.

[14] S. Karakuş and K. Demirci, “A-summation process and Korovkin-type approximation theorem for double sequences of positive linear operators," Mathematica Slovaca, (in press).

[15] P. P. Korovkin, "Linear operators and approximation theory," in Russian Monographs and Texts on Advanced Mathematics and Physics, Vol. III. New York/Delhi: Gordon and Breach Publishers, Inc./Hindustan Publishing Corp. (India), 1960, pp. vii+222.

[16] B. Kuttner, "On the Gibbs phenomenon for Riesz means," J. London Math. Soc., vol. 19, pp. 153-161, 1944. 
[17] F. Móricz and B. E. Rhoades, "Almost convergence of double sequences and strong regularity of summability matrices," Math. Proc. Cambridge Philos. Soc., vol. 104, no. 2, pp. 283-294, 1988.

[18] F. Móricz, "Statistical convergence of multiple sequences," Arch. Math. (Basel), vol. 81, no. 1, pp. $82-89,2003$.

[19] M. Mursaleen and O. H. H. Edely, "Statistical convergence of double sequences," J. Math. Anal. Appl., vol. 288, no. 1, pp. 223-231, 2003.

[20] M. Mursaleen and E. Savaş, "Almost regular matrices for double sequences," Studia Sci. Math. Hungar., vol. 40, no. 1-2, pp. 205-212, 2003.

[21] R. F. Patterson and E. Savaş, "Uniformly summable double sequences," Studia Sci. Math. Hungar., vol. 44, no. 1, pp. 147-158, 2007.

[22] A. Pringsheim, "Zur Theorie der zweifach unendlichen Zahlenfolgen," Math. Ann., vol. 53, no. 3, pp. 289-321, 1900 .

[23] C. Radu, “A-summability and approximation of continuous periodic functions," Stud. Univ. Babeş-Bolyai Math., vol. 52, no. 4, pp. 155-161, 2007.

[24] G. M. Robison, "Divergent double sequences and series," Trans. Amer. Math. Soc., vol. 28, no. 1, pp. 50-73, 1926.

[25] E. Savaş and B. E. Rhoades, "Double summability factor theorems and applications," Math. Inequal. Appl., vol. 10, no. 1, pp. 125-149, 2007.

Authors' addresses

\section{Sevda Karakuș}

Sinop University, Faculty of Sciences and Arts, Department of Mathematics, 57000, Sinop, Turkey

E-mail address: skarakus@sinop.edu.tr

\section{Kamil Demirci}

Current address: Sinop University, Faculty of Sciences and Arts, Department of Mathematics, 57000, Sinop, Turkey

E-mail address: kamildesinop.edu.tr 